\title{
Nutrition Education and Dietary Behavior Change Games: A Scoping Review
}

\author{
Tom Baranowski, PhD, ${ }^{1}$ Courtney Ryan, MS, RD, Andrés Hoyos-Cespedes, ${ }^{2}$ \\ and Amy Shirong Lu, $\mathrm{PhD}^{3,4}$
}

\begin{abstract}
Games provide an attractive venue for engaging participants and increasing nutrition-related knowledge and dietary behavior change, but no review has appeared devoted to this literature. A scoping review of nutrition education and dietary behavior change videogames or interactive games was conducted. A systematic search was made of PubMed, Agricola, and Google Scholar. Information was abstracted from 22 publications. To be included, the publication had to include a videogame or interactive experience involving games (a videogame alone, minigames inserted into a larger multimedia experience, or game as part of a human-delivered intervention); game's design objective was to influence dietary behavior, a psychosocial determinant of a dietary behavior, or nutrition knowledge (hereinafter referred to as diet-related); must have been reported in English and must have appeared in a professional publication, including some report of outcomes or results (thereby passing some peer review). This review was restricted to the diet-related information in the selected games. Diversity in targeted dietary knowledge and intake behaviors, targeted populations/audiences, game mechanics, behavioral theories, research designs, and findings was revealed. The diversity and quality of the research in general was poor, precluding a meta-analysis or systematic review. All but one of the studies reported some positive outcome from playing the game(s). One reported that a web-based education program resulted in more change than the game-based intervention. Studies of games may have been missed; a number of dietary/nutrition games are known for which no evaluation is known; and the data presented on the games and research were limited and inconsistent. Conclusions and Implications: A firmer research base is needed to establish the efficacy and effectiveness of nutrition education and dietary behavior change games.
\end{abstract}

Keywords: Games, Nutrition education, Dietary behavior change, Scoping

\section{Introduction}

D IETARY INTAKE HAS been related to heart disease, diabetes, several cancers, stroke, and perhaps adiposity. ${ }^{1}$ Dietary behavior change programs have been shown to change diet, but the mediating variables are not clear. ${ }^{2,3}$ Nutrition knowledge change programs (usually called nutrition education) by themselves have been shown to be inadequate to change behavior, ${ }^{4}$ but some knowledge is a likely precondition of effective behavior change. ${ }^{5}$ Dietary behavior change programs are needed that appeal to large numbers and diverse types of people who could benefit from the educational/behavior change procedures. Innovative approaches to dietary change are needed to engage participants in enjoyable experiences to reach the largest number of participants.

Electronic media are an increasingly popular channel for health promotion, meeting the needs and interests of modern populations. ${ }^{6-8}$ At least six types of electronic media for health promotion have been identified: web-based

\footnotetext{
${ }^{1}$ Department of Pediatrics, USDA/ARS Children's Nutrition Research Center, Baylor College of Medicine, Houston, Texas.

${ }^{2}$ Bouvé College of Health Sciences, Northeastern University, Boston, Massachusetts.

${ }^{3}$ Health Technology Lab, Department of Communication Studies, College of Arts, Media \& Design, Bouvé College of Health Sciences, Northeastern University, Boston, Massachusetts.

${ }^{4}$ Department of Health Sciences, Bouvé College of Health Sciences, Northeastern University, Boston, Massachusetts.
} 
educational/therapeutic programs; tailored motivational messaging systems; data collection and feedback systems; active videogames (also called exergames); diverse interactive multimedia; and diverse games. ${ }^{7}$ This review concerns games or interactive media incorporating games. A recent review of school nutrition education resources identified 14 of 32 curricula as incorporating games of one type or another to enhance learning, but provided no identification or analysis of the games. ${ }^{9}$ This suggests that games are increasingly recognized as an important learning tool, but the role and effectiveness of the games need to be analyzed. There has been recent interest in the use of "gamification," which incorporates aspects of games into the design of media interventions. ${ }^{10}$ Gamification most often has employed rewards, ${ }^{10}$ thereby not including some of the most interesting aspects of games, for example, repeated losing under the rules of the game to learn a new principle without real-world consequences, and are most appropriately reviewed when considering those behavior change procedures, ${ }^{11,12}$ not when reviewing the effects of games.

No consistent, commonly accepted concept exists for what constitutes a game. Games generally have rules that govern actions within the game with the possibility of winning and, importantly, losing based on the rules; feedback on play within the game (often points assigned); and a story or narrative (or an associated back story), among many other possible game elements. ${ }^{13}$ Games can provide attractive learning and behavior change media since they can be designed to provide fun, enjoyment, or engagement (thereby providing intrinsic motivation to play) ${ }^{14}$; a multisensory experience to facilitate different learning styles ${ }^{15}$; safe opportunities for drill and practice with feedback; training for higher-order skills, such as critical thinking, problem solving, team work, strategic thinking, and planning ${ }^{16}$; role playing for constructivist learning ${ }^{17}$; experimentation with new identities; development of social skills ${ }^{18}$; modeling of behaviors $^{19}$; training in self-regulation ${ }^{19}$; and/or immersion of players in a storyline into which behavior change procedures can be built. ${ }^{20}$

While several systematic reviews and/or meta-analyses have appeared on games for health, ${ }^{6,21-24}$ and even included a few nutrition education games as a subcomponent, no review has appeared devoted to the consideration of games for nutrition education or dietary behavior change alone. Some reviews have indicated that research on few nutrition games have appeared ${ }^{25}$; one found only four games $^{26}$; a review of digital interventions included only one nutrition education game, ${ }^{27}$ and another included only six. $^{28}$ This scoping review of the diet-related video or interactive games literature attempts to describe the current state of the art in a substantial number of studies involving nutrition education games and identify limitations in the research, which need to be remedied to advance this important intervention medium.

\section{Methods}

A protocol for this analysis was not preregistered due to the long idea-incubation period and a lack of clarity about what we might find in our literature search. The structure and sequence of this review follows the PRISMA guidelines, as possible. $^{29}$

\section{Eligibility criteria}

To be included in this review, the publication had to include a videogame or interactive experience involving games (i.e., the intervention was a videogame alone, minigames were inserted into a larger multimedia experience, or a game was part of a human-delivered intervention); the game's design objective was to influence dietary behavior, a psychosocial variable believed to be a determinant of a dietary behavior, or some aspect of nutrition knowledge (hereinafter referred to as diet-related); must have been reported in English and must have appeared in a professional publication, including some report of outcomes or results (thereby passing some peer review). No additional exclusionary criteria were employed. This review was restricted to the diet-related information in the selected games.

\section{Information sources}

Following the guidelines for a scoping review, ${ }^{30}$ this review included two search cycles. Both searches of the literature were conducted using PubMed, Agricola (the agricultural database, since the U.S. Department of Agriculture appeared to fund much of the early work on nutrition games), and Google Scholar, using the search terms (Nutrition AND Games) and (Diet AND Games).

\section{Search}

One author conducted the initial systematic search of the databases through December 31, 2016, while the first author culled the articles for whether a game was involved in the identified articles. A second cycle was performed during September 2017, including only the articles produced from January 1, 2017 to September 30, 2017.

\section{Study selection}

Since the method of game delivery varied by time, there was an initial interest in the historical development of games for nutrition education. An intensive effort was made to identify any article that concerned a game involving nutrition or diet. This preliminary, broad, exploratory search revealed articles on a substantial variety of games involving nutrition education, including card, board, video, and other delivery channels. One hundred eighty-five abstracts of articles were analyzed; duplicates were removed. Since many of these articles were not research-based (i.e., no evaluation of effects), the investigators narrowed the focus to include articles meeting the eligibility criteria uncovered through the initial ad hoc search. Two coders reviewed the abstracts of these articles; duplicate entries were removed; and selected articles were retrieved and abstracted. In the search process, a number of intervention articles were identified that used one or more multimedia formats, but did not include games, ${ }^{31-39}$ and thereby were not included in this review.

\section{Data collection process}

To ensure inter-rater reliability and to authenticate data extraction and interpretation quality, two independent reviewers systematically analyzed the abstracts. Final selection was based on a $92 \%$ agreement between reviewers. Data abstraction was started after both reviewers received coding training and 
performed a coding of $10 \%$ of the articles as a pilot for testing reliability. Differences were resolved by multiple, internal, indepth discussions. Following training, each coder reviewed and extracted data from the 22 articles separately. The first author provided the final determination for all variables.

\section{Data items}

Each row in Tables 1-3 concerns a different study. The studies were summarized in regard to first author, year, country, game name, the key goals and hypotheses, whether a story/narrative was included, game elements or mechanics, behavior change procedures and theories employed, setting, target group, method of delivery, research design, sample size, outcome measures (specifically subjectively reported and objective measures), and diet-related outcomes. Story, often considered a key game element, ${ }^{6}$ was coded as present when there were characters other than the player, a logically sequenced set of events (storyline or arc), with the story ending and the outcome depending on game play. Game elements or mechanisms were abstracted as possible from the reports provided.

\section{Risk of bias in individual studies}

Quality of study or research bias items from the Cochrane Collaboration Risk of Bias Tool (CCRBT) and the Effective Public Health Practice Project Quality Assessment Tool (EPHPP $)^{40}$ were applied to all studies. Table 3 reports on the risk of bias/quality of research characteristics of each study, including whether the method of randomization was reported; randomization was concealed at baseline; prior power calculations were conducted; assessors were blinded; completeness of follow-up reported; selection bias reported; validity or reliability of the measures reported; possible confounding variables were accounted for in the analyses; and the trial protocol was preregistered.

\section{Synthesis of results}

Many of the included studies were pilot, feasibility or proof-of-concept studies with small samples, weak designs, and inconsistent and inadequate assessment, thereby precluding a meta-analysis or systematic review. Thus, the major value of this scoping review is to report the breadth of target groups, objectives and game mechanics, and identify specific research enhancements necessary to document the value of the gaming approach to diet-related change.

\section{Results}

\section{Study selection}

One hundred eighty-five articles were identified in the preliminary search: 61 from Agricola and 124 from PubMed. An additional 50 articles were identified in the second cycle. Twenty-two articles were identified on nutrition education or dietary change video or interactive games, hereinafter referred to as game.

\section{Study characteristics}

The first game article identified was published in 2001, with the number per year steadily increasing to eight in 2015-2017.

\section{Risk of bias within studies}

The quality or research bias of individual trials was poor, and is summarized in Table 3. Eleven of the 22 articles (50\%) were randomized clinical trials (RCTs), and $3(14 \%)$ were group cluster RCTs. Four (18\%) were single-group pre-post designs; two (9\%) were not randomized control designs, one was a cross-over trial, and one was an A-B-A-B reversal design. The method of randomization, concealment of randomization at baseline, and trial preregistration was reported by none of these trials (Table 3). Five (23\%) reported statistical power calculations before the conduct of the trial. While blinding of participants is not possible in behavior change trials, blinding of assessors is possible and was reported in only one trial.

Completeness of follow-up (withdrawals, dropouts) was reported in 12 trials $(55 \%)$. Selection bias (sample representativeness and participation rates) were reported by five trials $(23 \%)$. Validity and/or reliability of the measures was reported by 14 trials (64\%). Accounting for possible confounders of outcomes was reported by nine trials $(41 \%)$. Whether the investigators reported analyses of all the prespecified outcomes could not be assessed since none of the research protocols were preregistered.

\section{Results of studies}

Only one of the games reported no changes from playing the game on behavior or its determinants, ${ }^{41}$ but only $27.6 \%$ of the students in that study played the game. All the other studies reported some positive outcomes from game play, but few studies identified a primary outcome, and none identified what percentages of all possible or expected outcomes the games impacted. Another study revealed that a web-based education program was more effective than the game-based education. ${ }^{42}$ Most changes were relatively small, but few effect sizes were reported.

\section{Synthesis of results}

Thirteen of these games (59\%) targeted children at school, three $(14 \%)$ targeted children at home, three $(14 \%)$ in a laboratory, one any place, and only two (5\%) targeted adults. The sample sizes varied from 1876 to 20 . There were 14 (64\%) individual or group randomized clinical trials. The games were developed and tested largely in the United States $(n=13 ; 59 \%)$, Europe $(n=8 ; 36 \%)$, or Asia $(n=1)$.

A story (or narrative) was specifically included in 15 games $(68 \%)$. The other game elements or game mechanics varied enormously. A number of well-known popular existing game formats/mechanics should have been well known to the target audience (thereby minimizing the need to learn new game rules), for example, the concentration memory game, and adapted these game elements to achieve education purposes or influence behavior.

Five of these games (23\%) could be categorized more as primarily targeting some aspect of nutrition knowledge, while nine other games $(41 \%)$ could be categorized more as targeting dietary behavior change, and eight (36\%) as primarily targeting some combination of dietary psychosocial variables and behavior. A diverse variety of educational and/ or behavioral change theories with attendant diversity in the corresponding knowledge or behavior change procedures were identified as providing the conceptual foundation and 


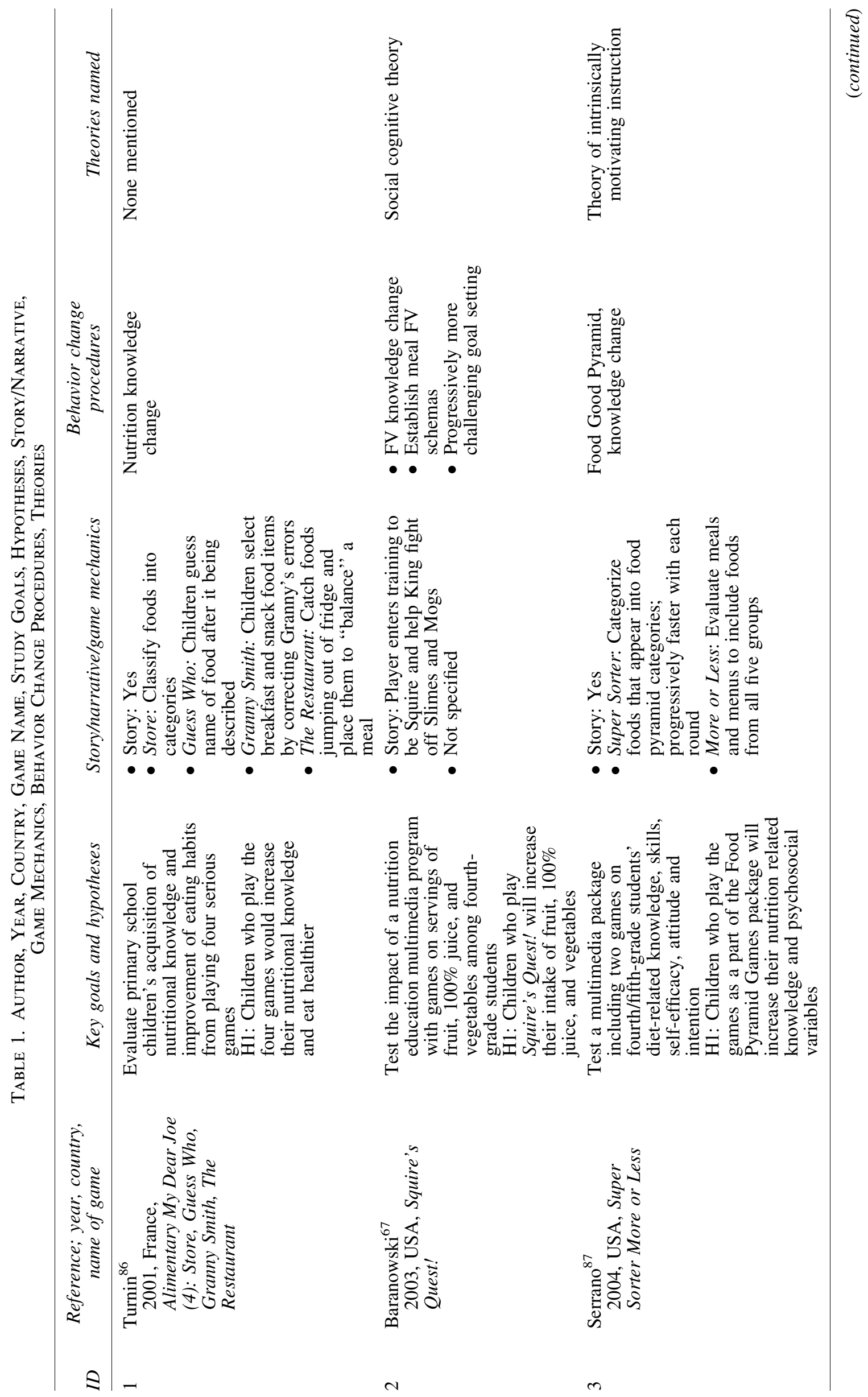




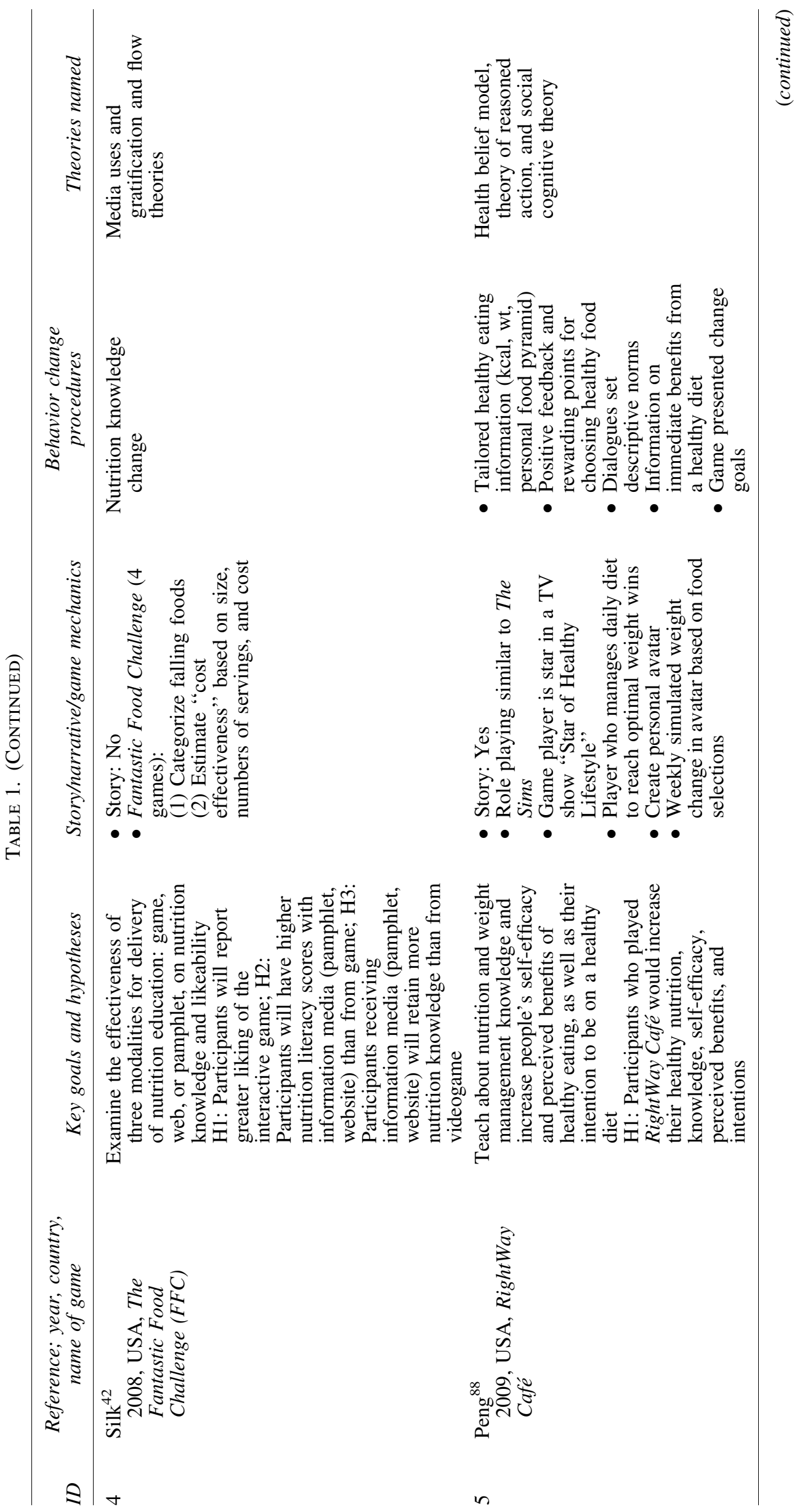




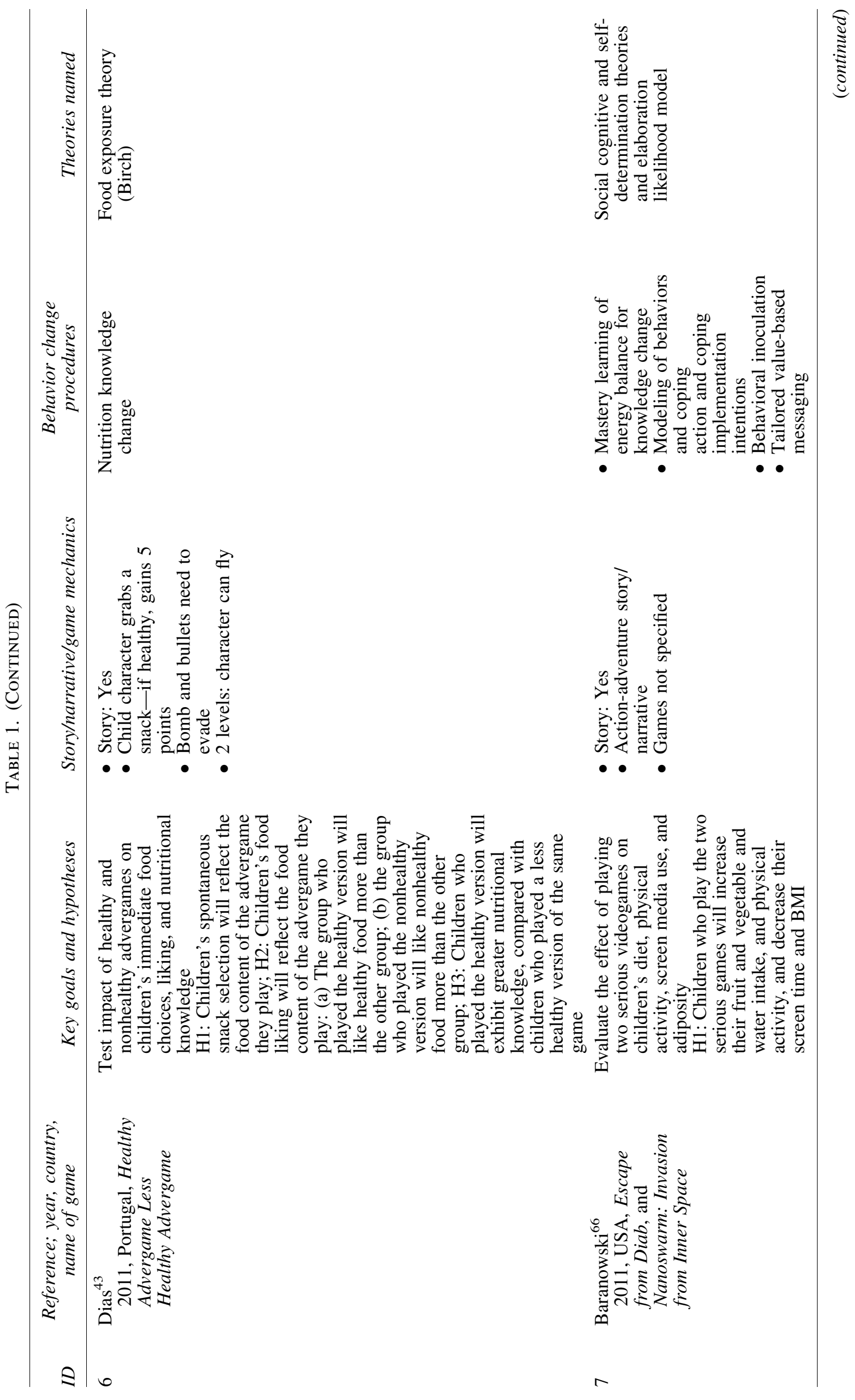




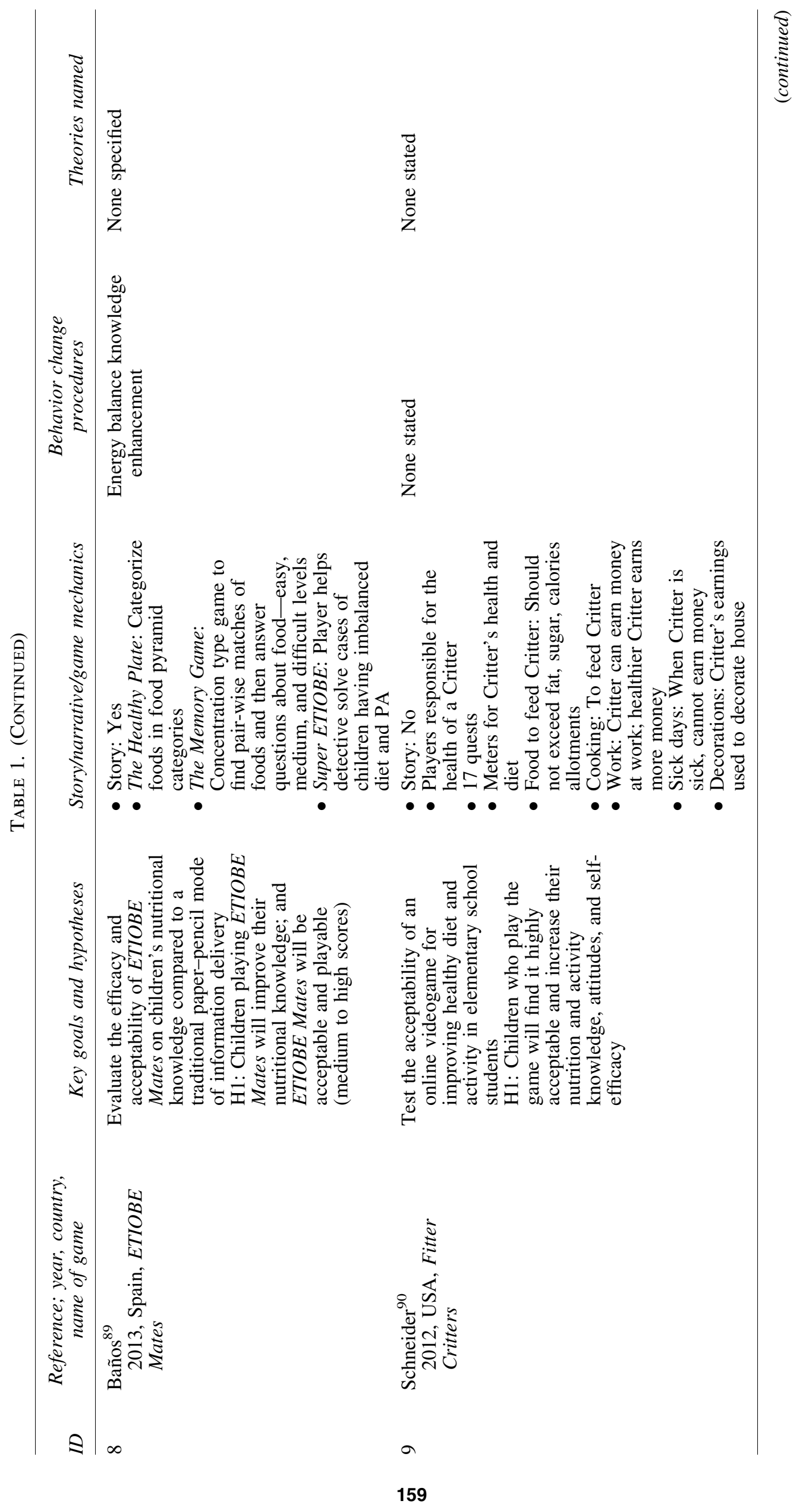




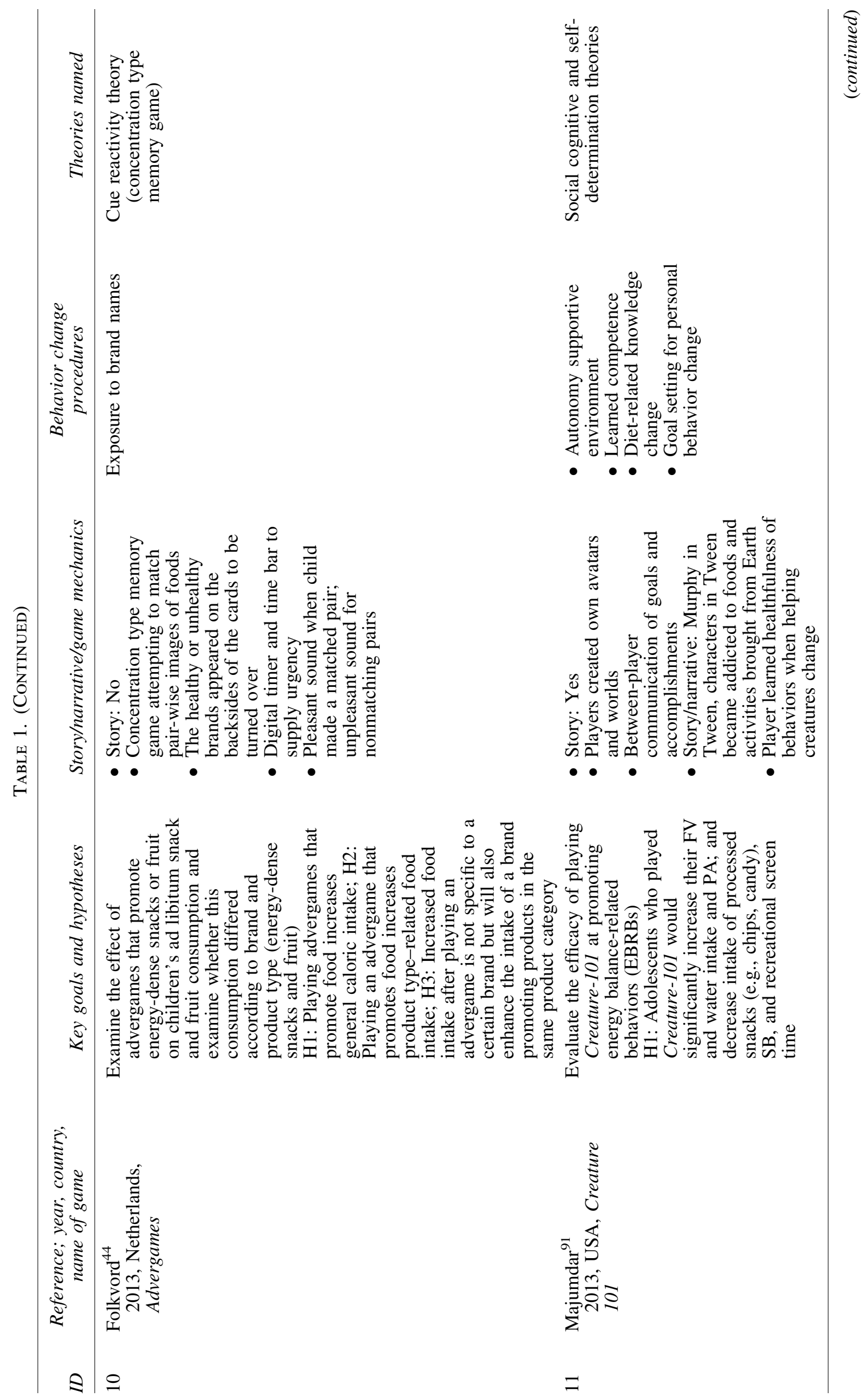




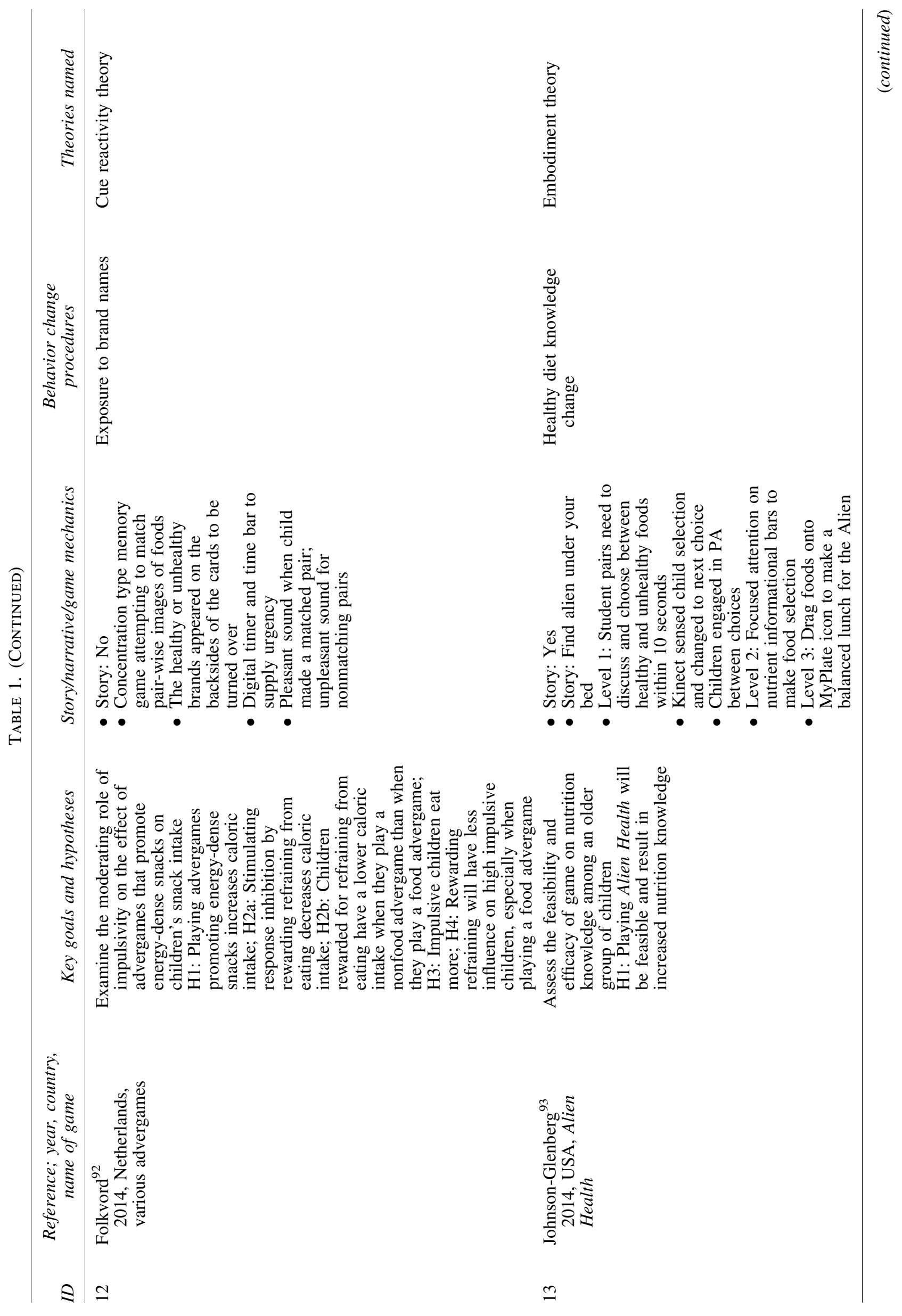




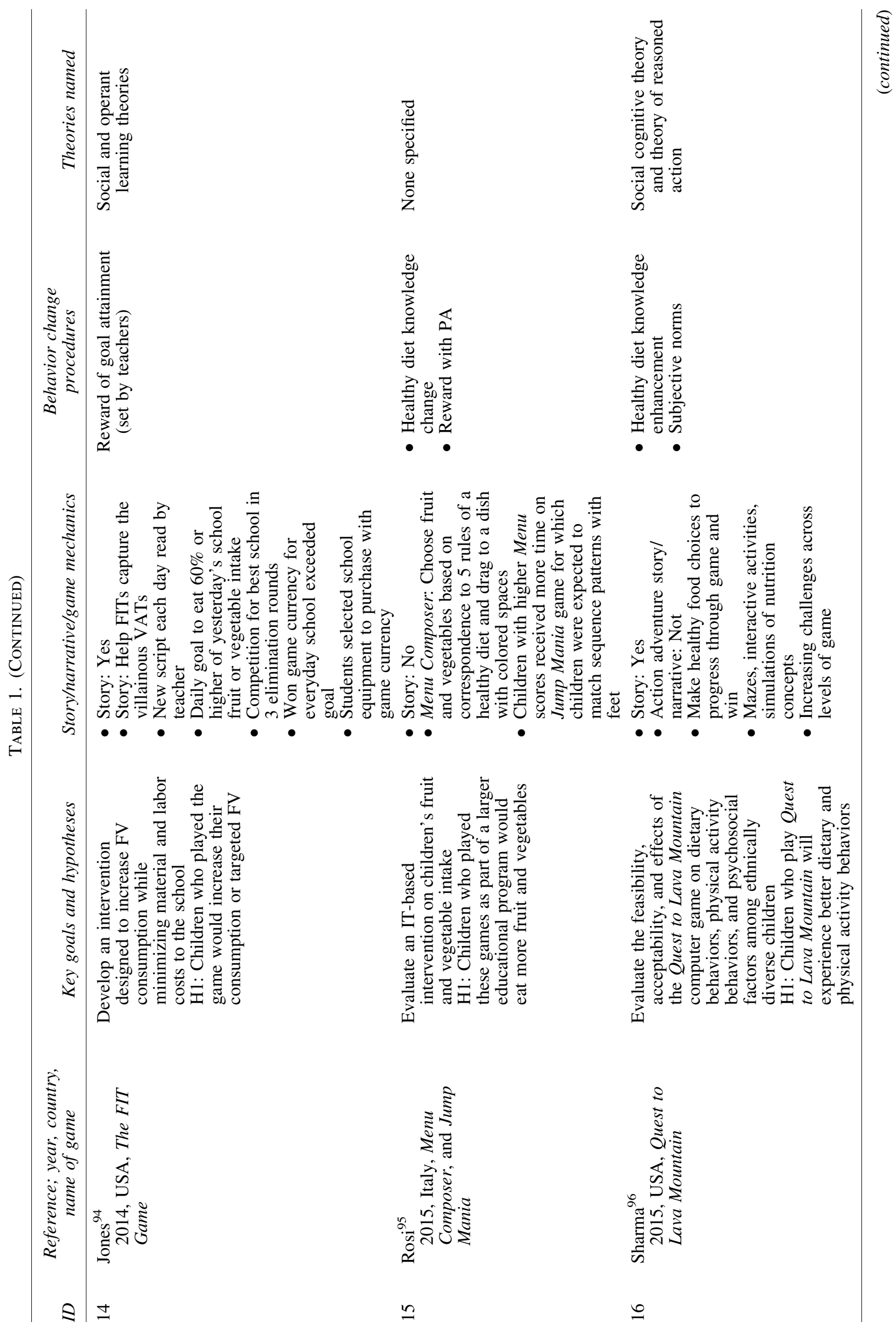




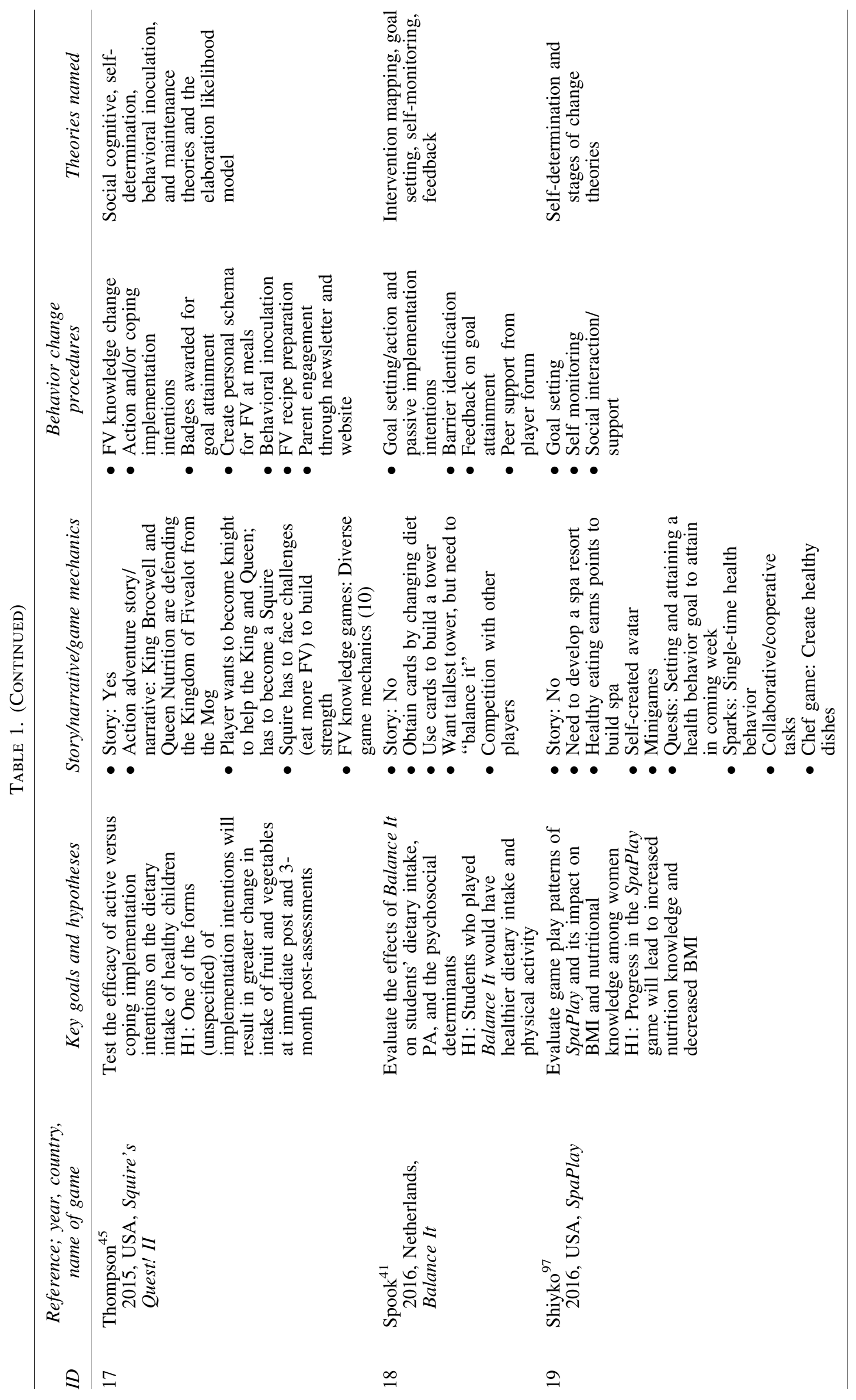




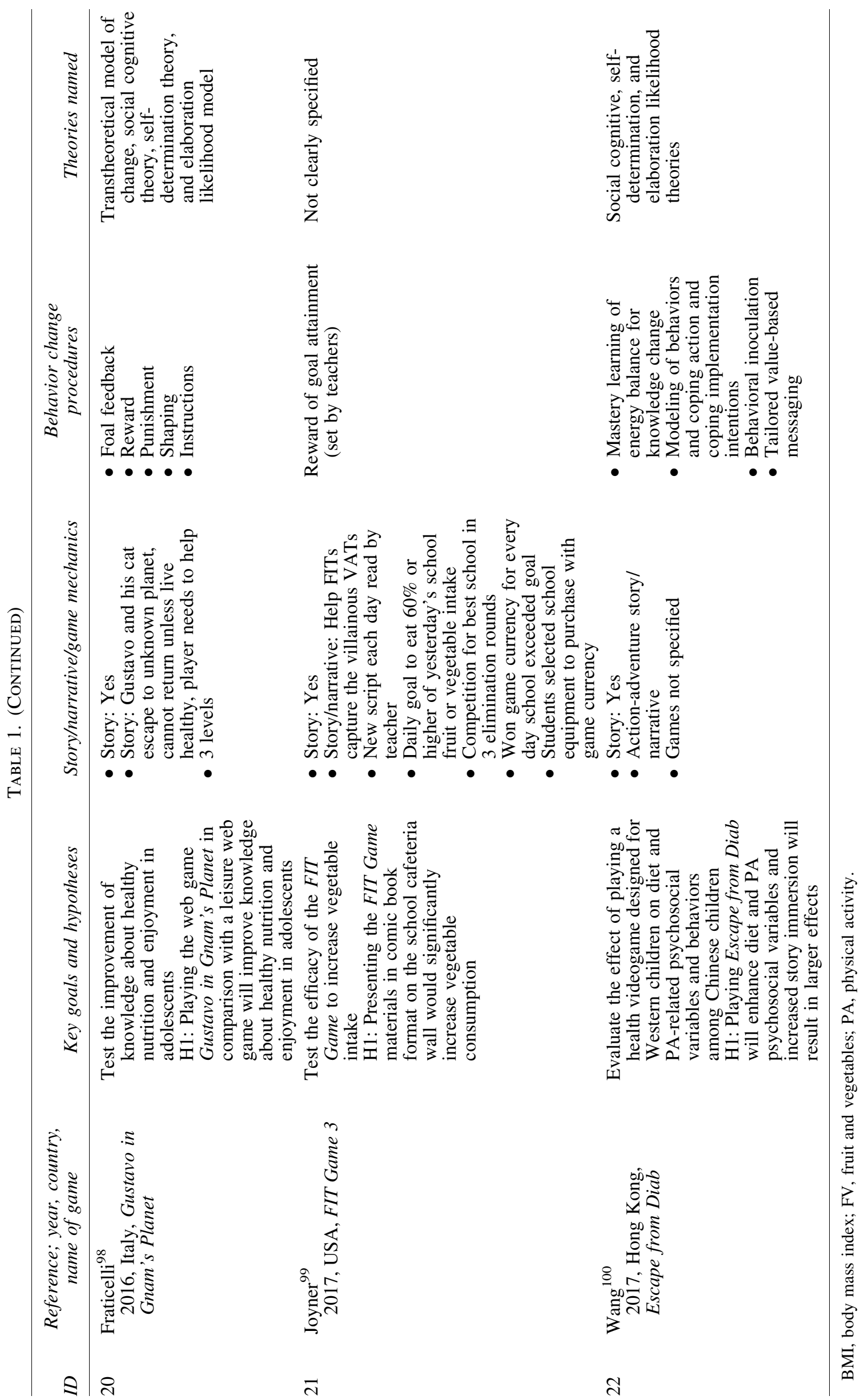




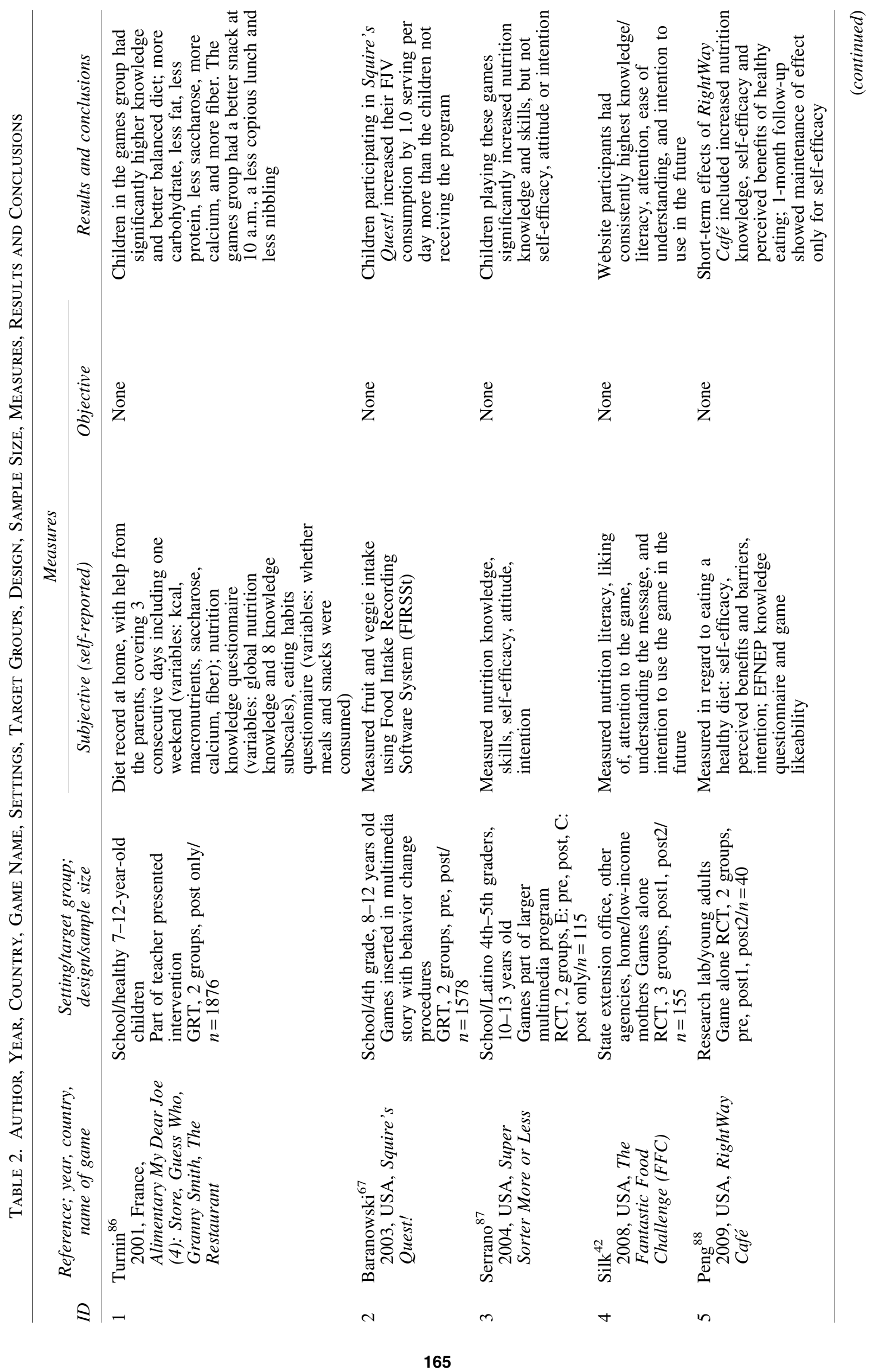




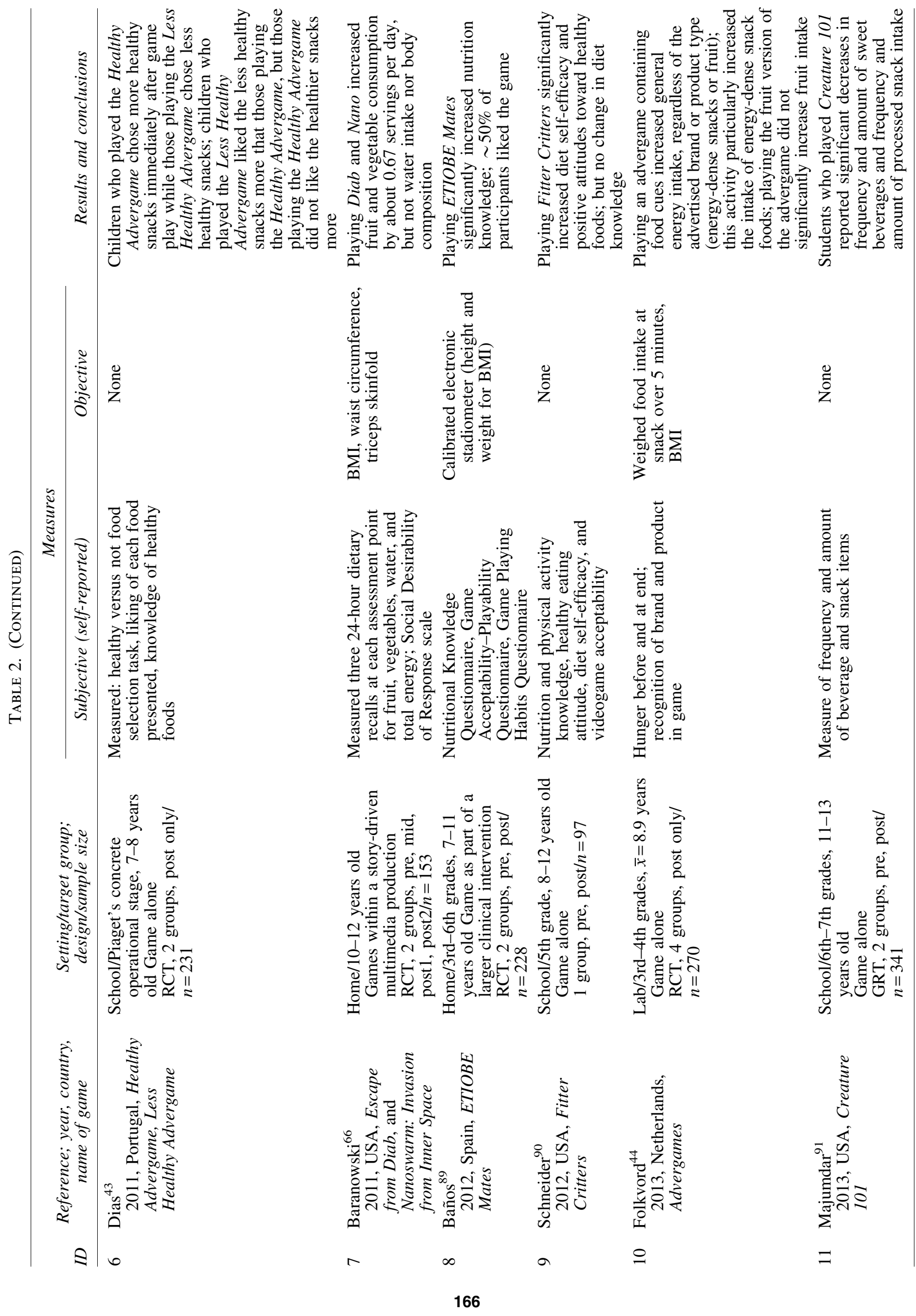




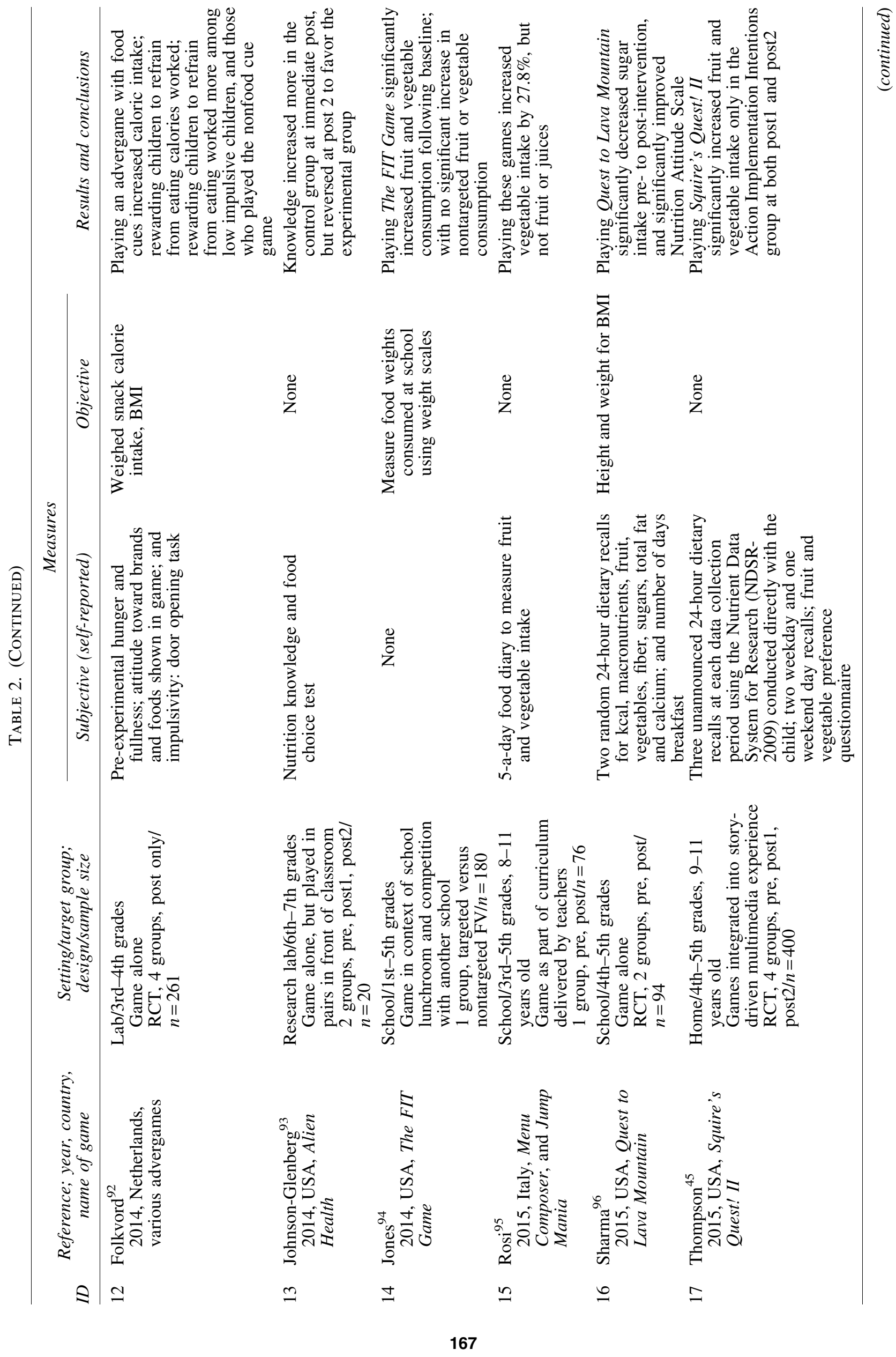




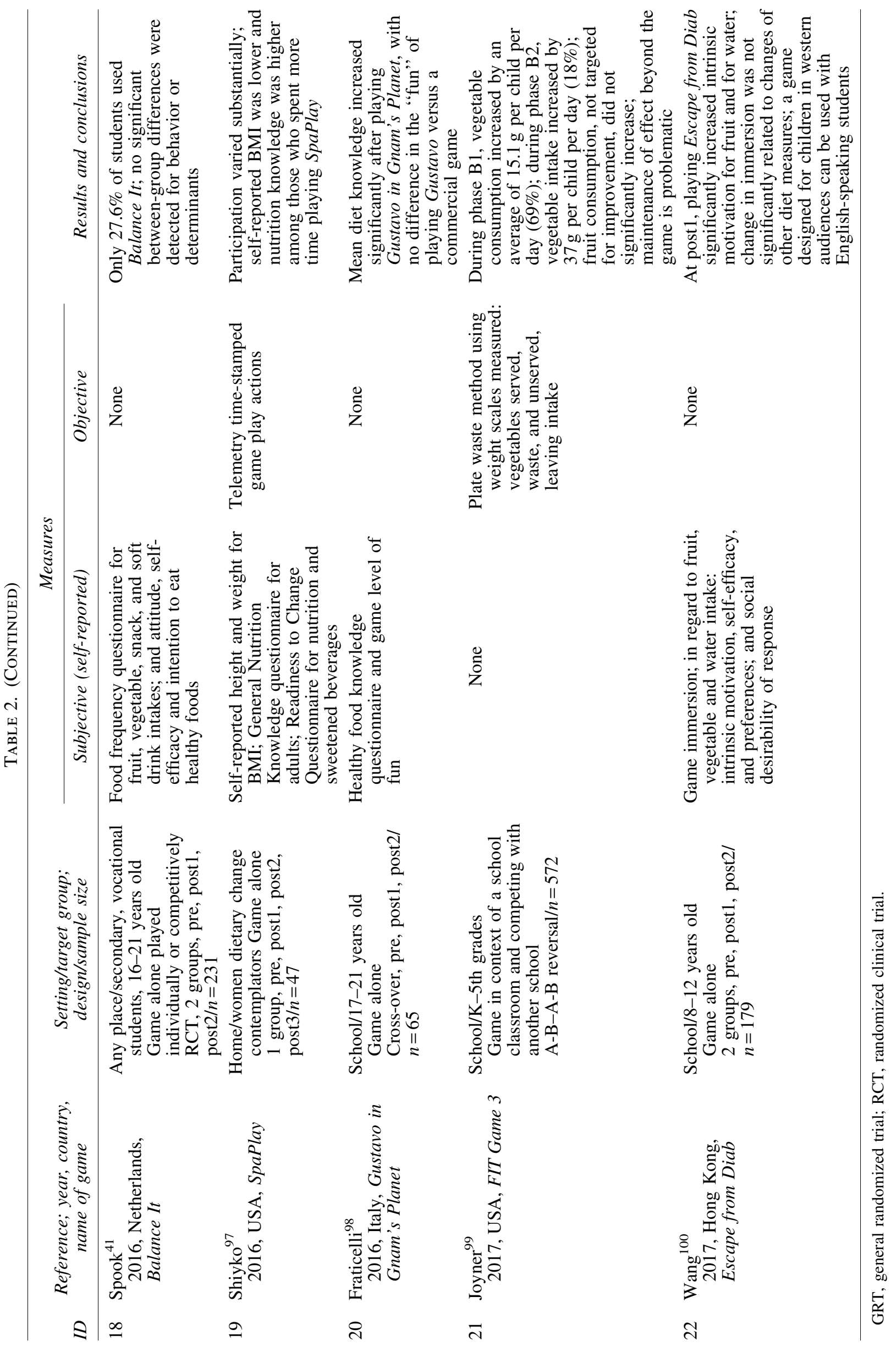




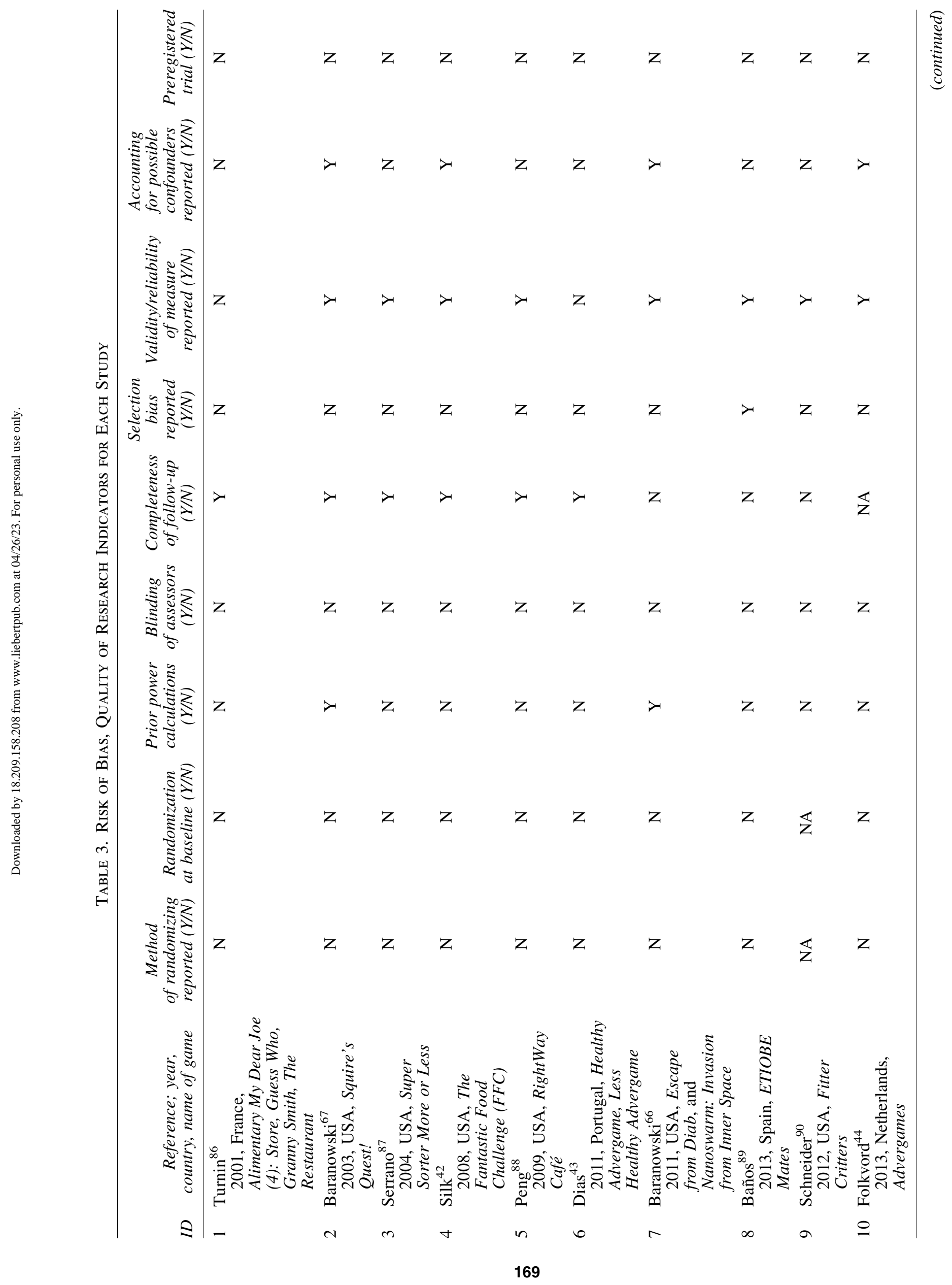




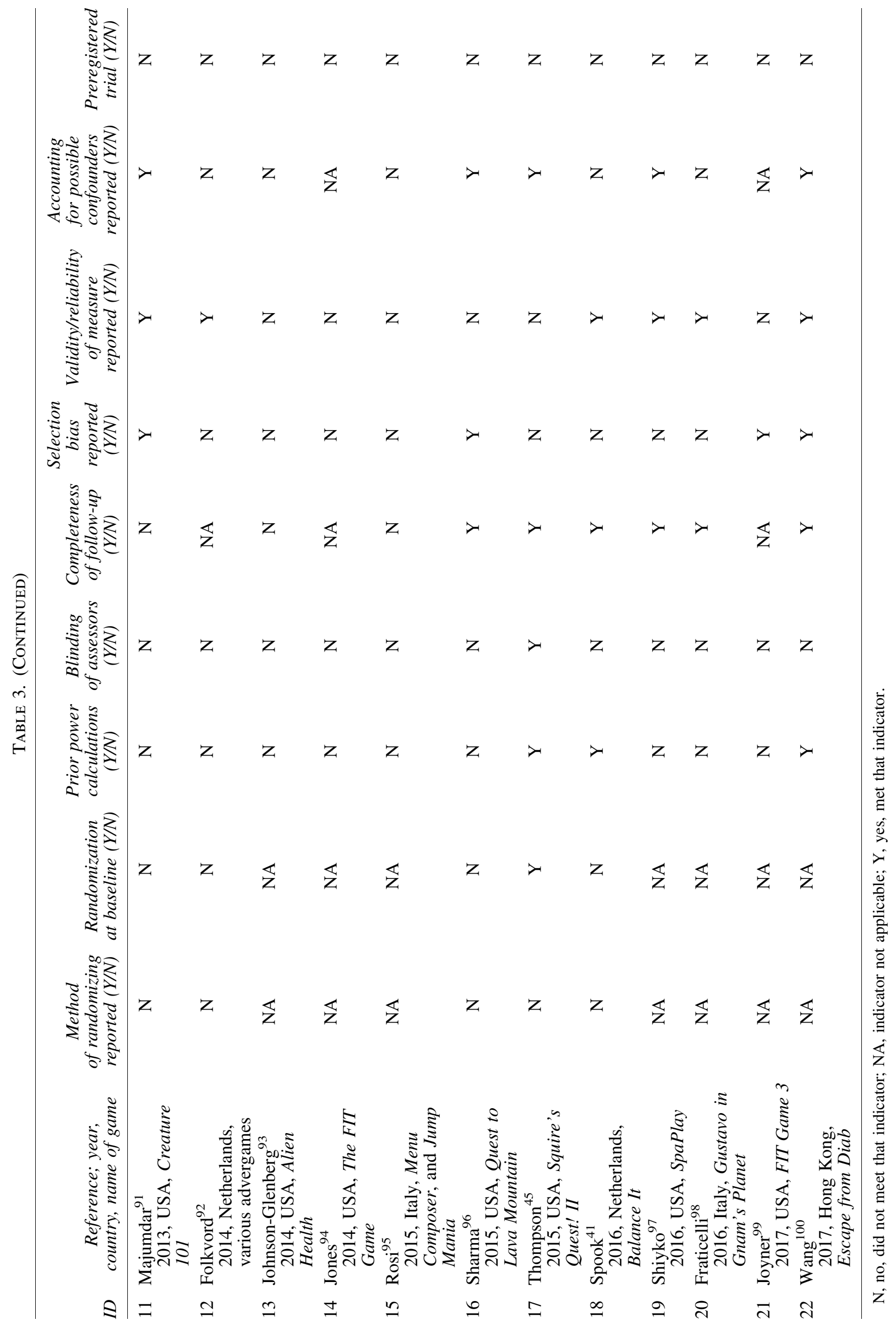


operationalization of the theory constructs for the game. Social cognitive theory appears to have been the most commonly stated theory, and nutrition knowledge change was the most commonly mentioned behavior change procedure.

The measures used to assess outcomes were perhaps even more diverse wherein different measures were often employed to assess the same knowledge or behavior construct across studies. Five studies $(23 \%)$ reported more objective methods of dietary intake assessment (e.g., weighed plate waste): three of these as part of laboratory-based tests of the effects of diet-related advergames, and two as part of a test of game play in a school lunchroom/cafeteria.

Three articles studied the effects of food advergames ${ }^{43,44}$ and showed that exposure to only 5 minutes of game play influenced the child's dietary intake immediately following the game. This suggests the possible strong (at least shortterm) behavioral impacts of dietary game play.

One game systematically varied two forms of a behavior change procedure, action and coping implementation intentions, and tested impact on fruit and vegetable intake within a four-group RCT. ${ }^{45}$ Since the programming keeps all other aspects of game play comparable, this is an ideal method for conducting tests of behavior change procedures for use in videogames, and perhaps outside of games.

\section{Discussion}

\section{Summary of the evidence}

While all but one of the articles reported some positive outcomes from playing the game(s), the quality of much (but not all) of the research reported left much to be desired, making it difficult to determine the extent to which games had the intended, and reported, effects. The research on dietrelated change games diverged in regard to target populations, dietary behavior targets (e.g., fruit, vegetables, general nutrition information, Mediterranean diet), theories (socialcognitive, persuasion, health belief model, attachment, socioconstructivist, or even none), game elements/mechanics, behavior change procedures, research designs, and measures. As a result, a meaningful meta-analysis or systematic review was not possible across all these studies at this time.

\section{Comparisons to other reviews}

A recent review of advergames alone indicated a small effect increasing unhealthy food intake. ${ }^{46}$ While that was not the primary focus of the current review, it does indicate that games can influence dietary intake to some extent under some circumstances. The review, including six games for health, noted the generally positive effects on nutrition knowledge, but the diversity in delivery, poor measurement of process variables and engagement, and thereby the inability to draw conclusions about effects. ${ }^{28}$ We noted similar patterns, but in a larger number of nutrition education game studies, and provided a more detailed assessment of the limitations in the research methods.

\section{Future directions/needed research}

Conceptual frameworks exist to guide the development of sophisticated G4H (Games for Health). ${ }^{47}$ Research from three large behavior change trials (not using games) used mediating variable analyses to validate a multi-theoretical model to specify the conceptual framework for the design of health behavior change interventions that are likely to be effective. ${ }^{48}$ Several conceptual models have been advanced to account for how games may influence diet-related outcomes. ${ }^{49-51}$ Some of these models interrelate game elements and behavior change principles, ${ }^{50,51}$ while others emphasize the relevance of food cues and individual predisposing factors (e.g., impulsivity). ${ }^{49}$ Little research, however, has tested aspects of these models with games. Much of the effort in diet-related games has been to design, develop, and test a game or interactive experience including game(s) to influence outcomes, rather than test principles of game mechanics or behavior-mediating variables within games. Research is urgently needed that systematically varies aspects of game mechanics or behavior change procedures within games to optimize their effects on game engagement, ${ }^{52}$ maintenance of interest, psychosocial mediators of change, and behavior change (shorter or longer terms). Thompson et al. systematically varied four types of goal setting/implementation intentions within Squire's Quest! and demonstrated that action implementation intention was the only procedure to maintain changes 3 months after the end of the game. ${ }^{45}$ This research provides an important model for making future contributions to an understanding of how game mechanics can influence change in behavior.

No commonly accepted compendium or taxonomy of elements in games exists. The "game lenses" identified by Schell ${ }^{13}$; the "playful experience cards" of Bjork and Holopainen $^{53}$; and/or Lucero et al.'s playful experiences list ${ }^{54}$ preliminarily enumerate items for consideration. Without a commonly accepted compendium, consistencies in game mechanics across games may have been missed since different words may have been used to specify an essentially common underlying mechanic or set of mechanics. Developing and obtaining consensus for such a taxonomic compendium should be a high priority to permit the identification and comparison of game elements in future systematic reviews and meta-analyses and also to identify mechanics that are most engaging. Such taxonomic compendia would also facilitate the conduct of process evaluations, ${ }^{55}$ which relate participation in game elements to outcomes.

A compendium of behavior change procedures has been proposed, ${ }^{56}$ although challenges have been reported in classifying procedures into the proposed categories. ${ }^{57}$ Such a common taxonomy should also be used in games research to clearly specify what procedures were employed. Of course the same procedure (e.g., goal setting) could be implemented in a variety of ways in the context of a game, ${ }^{58}$ with each variant possibly affecting outcome in a different way, indicating specific details of the intervention design need to be presented in the reported outcome or process evaluations.

Fifteen games $(68 \%)$ incorporated a story or narrative in one form or another. Given the potential for story immersion to influence attention to change procedures (especially behavior change messaging), ${ }^{20}$ developing and incorporating stories into nutrition education games may be a way to enhance effectiveness. Alternatively, the incorporation of a story into a game may distract the player from active game play, thereby reducing player game enjoyment. ${ }^{59}$ Several stories have been tested and demonstrated to change behavior without a game. ${ }^{36,60}$ A study that designs and compares separate story and game elements with their integration 
would be an important contribution to our knowledge of nutrition education games (and games in general).

There seems to be an implicit assumption that simply providing a diet-related change program in a game format will automatically make the activity fun, or engaging, and thereby motivate the player to substantially increase the knowledge or change the behavior at substantial levels. Alternatively, one of the studies revealed that a web-based education program was more effective that a game-based program. ${ }^{42}$ Unfortunately, the relative quality of these two programs cannot be meaningfully assessed from the published information, and thus it is not clear if primarily the medium (web-based or game-based) or qualities of the programs influenced this outcome. The modest changes in knowledge and behavior that have been reported in these studies suggest the assumption of game dominance is not well supported. The role of "fun" in games and game play has not been well specified; the definition and measurement of fun or enjoyment has not been clearly delineated ${ }^{61}$; story or narrative may contribute to the fun of games, but this has not been clearly assessed. ${ }^{62}$ If fun in games was better understood, games might be designed to enhance the fun aspects to, in turn, enhance the dietary change.

Many of these games targeted nutrition knowledge, often as the primary or only target. Each set of authors appears to have created their own measure of nutrition knowledge with unknown psychometric properties. While knowledge for academic purposes (e.g., types of dietary fats; how sodium, potassium, and magnesium interact to influence blood pressure) can be valuable, all these games were targeted in one way or another to positively impact health, often through dietary behavior change. The field would benefit from a library of nutrition knowledge items that tapped information that were preconditions for behavior change (e.g., how to make a salad) and could be incorporated into computer adaptive testing ${ }^{63}$ to provide brief measures of outcomes comparable across studies.

Developing a diet-related intervention that can be expected to impact body mass index (perhaps the most prevalent nutrition-related health problem) for obesity prevention requires that the game impact the necessary relevant knowledge and psychosocial variable(s) (e.g., knowledge, self-efficacy, intrinsic motivation) to change the targeted behavior(s) (e.g., diet, physical activity, sedentary behavior, and/or sleep) for a long enough time to change body composition. ${ }^{64}$ There are few examples of such large games. The time to develop and evaluate them can take years, ${ }^{65,66}$ and the cost for their development can be staggering (millions of dollars). It seems unlikely that this level of funding will become generally available for game development anytime soon. Rather than creating a single integrated multimedia experience that incorporates games to address dietary change, it may be appropriate to develop a comprehensive intervention structure (including multimedia and face-to-face components) into which a series of games could be developed and inserted to achieve those outcomes for which games are most effective, for example, knowledge enhancement, simulation of interpersonal interactions, etc. Alternatively, dietary behavior change requires learning some new skills that could be learned through games, for example, food preparation, ${ }^{67}$ menu planning, effective food parenting practices, ${ }^{68}$ and even taste testing. Games attempting to increase these skills or behaviors could be developed, tested, and incorporated into larger behavior change programs.

There has been substantial concern about the quality of research, also called risk of bias, in general ${ }^{69-74}$ and how to incorporate issues of quality into systematic reviews. ${ }^{75,76}$ The general quality of the evidence in support of games on nutrition knowledge or behavior change outcomes ${ }^{77}$ was low and needs further enhancement. This has been pointed out elsewhere. ${ }^{78,79}$ Only five of these nutrition education game studies used objective measures of dietary intake to assess dietary change outcomes. Subjective measures tend to be subject to bias (i.e., low validity or accuracy) and unreliability (or imprecision). ${ }^{80}$ Both active and passive methods of more objective dietary intake assessment ${ }^{81}$ are being developed and refined for use in uncontrolled community settings and should be considered for future use as measures of outcome of games for nutrition education. $\mathrm{G} 4 \mathrm{H}$ researchers need to expend substantially more effort to enhance the quality of this research if for no other reason than selfinterest, that is, to increase the likelihood of obtaining funding for future research. ${ }^{82}$

Many of the studies identified a substantial number of behavioral, psychosocial, and/or anthropometric or physiological outcomes, without specifying a primary outcome. This approach to evaluation runs a high risk of type 1 statistical error (i.e., obtaining a statistically significant effect purely by chance). ${ }^{83,84}$ Diet-related game research should specify a primary outcome upon which power calculations are conducted, with prespecified hypotheses or models of the way in which game play is expected to influence the outcome. ${ }^{85}$ In this way, tests can be made of whether the game influenced the targeted variable(s) as designed. The stronger our knowledge base about how games result in change, the more effective ensuing games can be if designed based on what has been learned.

\section{Limitations}

One or more game studies may have been missed. A number of diet-related games have been available for purchase, but about which nothing has been published. (At least one game developer has been overheard saying "I have all the evaluation I want or need. My games sell."') A recent systematic review of G4H identified a total of 1743 games produced between 1983 and 2016, of which 99 (6.4\%) were about nutrition education and eating disorders. ${ }^{8}$ The number of academic publications that evaluated the efficacies of those games is far less than the games produced. This review was based on reports of games in publications that tended to provide minimal details, which could have resulted in mischaracterizing the games or studies. On one hand, we may miss existing games that were effective but never evaluated. On the other hand, actual play of the games might have provided different characterizations and insights, but many of the games no longer function on modern technology.

\section{Conclusion}

To the best of our knowledge, this is the first English review devoted to diet-related video or interactive games. Although a variety of diet-related games have been reported, the research base is inadequate to ascertain whether putting nutrition education or diet-related behavior change into a 
game format consistently results in the desired outcomes, or what aspects of game elements or behavior change procedures appear to be most effective. Future diet-related game research should use randomized controlled designs; common validated outcome measures (preferably more objective measures); behavior change procedures as specified in recent taxonomic inventories; and be guided by conceptual models that reflect the complexities of game design, user experience, and their interactivity. A taxonomic inventory of game elements needs to be developed to clearly specify game designs, and facilitate game reporting and comparisons of outcomes. Research is needed that systematically varies game elements between different versions of the same game to test what game elements produce the most change. Different concepts of fun in game play need to be comparatively tested. Perhaps we need a leaderboard of game researchers and their numbers of publications weighted by the quality of their contributions to make a game of it.

\section{Acknowledgments}

This work is a publication of the U.S. Department of Agriculture (USDA/ARS) Children's Nutrition Research Center, Department of Pediatrics, Baylor College of Medicine, Houston, Texas, and had been funded in part with federal funds from the USDA/ARS under Cooperative Agreement No. 58-3092-5001. The authors thank Harley Edge for helping with article selection and coding process.

\section{Author Disclosure Statement}

No competing financial interests exist.

\section{References}

1. Boeing H, Bechthold A, Bub A, et al. Critical review: Vegetables and fruit in the prevention of chronic diseases. Eur J Nutr 2012; 51:637-663.

2. Teixeira PJ, Carraca EV, Marques MM, et al. Successful behavior change in obesity interventions in adults: A systematic review of self-regulation mediators. BMC Med 2015; 13:84.

3. Diep CS, Chen TA, Davies VF, et al. Influence of behavioral theory on fruit and vegetable intervention effectiveness among children: A meta-analysis. J Nutr Educ Behav 2014; 46:506-546.

4. Contento I, Balch GI, Bronner YL, et al. The effectiveness of nutrition education and implications for nutrition education policy, programs, and research: A review of research. J Nutr Educ 1995; 27:277-418.

5. Bandura A. Social cognitive theory: An agentic perspective. Annu Rev Psychol 2001; 52:1-26.

6. Baranowski T, Buday R, Thompson DI, et al. Playing for real: Video games and stories for health-related behavior change. Am J Prev Med 2008; 34:74-82.

7. Baranowski T, Frankel L. Let's get technical! Gaming and technology for weight control and health promotion in children. Child Obes 2012; 8:34-37.

8. Lu AS, Kharrazi H. A state-of-the-art systematic content analysis of games for health. Games Health J 2018; 7:1-15.

9. Peralta LR, Dudley DA, Cotton WG. Teaching healthy eating to elementary school students: A scoping review of nutrition education resources. J Sch Health 2016; 86:334345.
10. Sardi L, Idri A, Fernandez-Aleman JL. A systematic review of gamification in e-Health. J Biomed Inform 2017; $71: 31-48$.

11. Corepal R, Tully MA, Kee F, et al. Behavioural incentive interventions for health behaviour change in young people (5-18 years old): A systematic review and meta-analysis. Prev Med 2018; 110:55-66.

12. Lewis ZH, Swartz MC, Lyons EJ. What's the point?: A review of reward systems implemented in gamification interventions. Games Health J 2016; 5:93-99.

13. Schell J. The Art of Game Design, A Book of Lenses, 2nd ed. Boca Raton, FL: CRC Press; 2015.

14. Mellecker R, Lyons EJ, Baranowski T. Disentangling fun and enjoyment in exergames using a expanded design, play, experience framework: A narrative review. Games Health J 2013; 2:142-149.

15. Castelhano N, Silva F, Rezende M, et al. Ludic content in multisensory stimulation environments: An exploratory study about practice in Portugal. Occup Ther Int 2013; 20: 134-143.

16. Nouchi R, Taki Y, Takeuchi H, et al. Brain training game boosts executive functions, working memory and processing speed in the young adults: A randomized controlled trial. PLoS One 2013; 8:e55518.

17. Levitt C, Adelman DS. Role-playing in nursing theory: Engaging online students. J Nurs Educ 2010; 49:229-232.

18. Granic I, Lobel A, Engels RC. The benefits of playing video games. Am Psychol 2014; 69:66-78.

19. Baranowski T, Thompson D, Buday R, et al. Design of video games for children's diet and physical activity behavior change. Int J Comp Sci Sport 2010; 9:3-17.

20. Lu AS, Baranowski T, Thompson D, et al. Story immersion of videogames for youth health promotion: A review of literature. Games Health J 2012; 1:199-204.

21. DeSmet A, Van Ryckeghem D, Compernolle S, et al. A meta-analysis of serious digital games for healthy lifestyle promotion. Prev Med 2014; 69:95-107.

22. Guy S, Ratzki-Leewing A, Gwadry-Sridhar F. Moving beyond the stigma: Systematic review of video games and their potential to combat obesity. Int J Hypertens 2011; 2011:179124.

23. Kharrazi H, Lu AS, Gharghabi F, et al. A scoping review of health game research: Past, present, and future. Games Health J 2012; 1:153-164.

24. Lu AS, Kharrazi H, Gharghabi F, et al. A systematic review of health videogames on childhood obesity prevention and intervention. Games Health J 2013; 2:131-141.

25. Khanana K, Law ELC. Designing children's digital games on nutrition with playability heuristics. In: CHI'13 Extended Abstracts on Human Factors in Computing Systems. Paris, France: ACM; 2013, pp. 1071-1076.

26. do Amaral E Melo GR, de Carvalho Silva Vargas F, Dos Santos Chagas CM, et al. Nutritional interventions for adolescents using information and communication technologies (ICTs): A systematic review. PLoS One 2017; 12:e0184509.

27. Rose T, Barker M, Maria Jacob C, et al. A systematic review of digital interventions for improving the diet and physical activity behaviors of adolescents. J Adolesc Health 2017; 61:669-677.

28. Nour M, Yeung SH, Partridge S, et al. A narrative review of social media and game-based nutrition interventions targeted at young adults. J Acad Nutr Diet 2017; 117:735752.e10. 
29. Moher D, Liberati A, Tetzlaff J, et al. Preferred reporting items for systematic reviews and meta-analyses: The PRISMA statement. PLoS Med 2009; 6:e1000097.

30. Colquhoun HL, Levac D, O'Brien KK, et al. Scoping reviews: Time for clarity in definition, methods, and reporting. J Clin Epidemiol 2014; 67:1291-1294.

31. Cullen KW, Thompson D, Chen TA. Outcome evaluation of Family Eats: An eight-session web-based program promoting healthy home food environments and dietary behaviors for African American families. Health Educ Behav 2017; 44:32-40.

32. Chomutare T, Johansen SG, Hartvigsen G, et al. Serious game co-design for children with type 1 diabetes. Stud Health Technol Inform 2016; 226:83-86.

33. Leung MM, Green MC, Cai J, et al. Fight for Your Right to Fruit: Development of a manga comic promoting fruit consumption in youth. Open Nutr J 2015; 9:82-90.

34. Domhardt M, Tiefengrabner M, Dinic R, et al. Training of carbohydrate estimation for people with diabetes using mobile augmented reality. J Diabetes Sci Technol 2015; 9: 516-524.

35. Ruggiero L, Moadsiri A, Quinn LT, et al. Diabetes Island: Preliminary impact of a virtual world self-care educational intervention for African Americans with type 2 diabetes. JMIR Serious Games 2014; 2:e10.

36. Bravender T, Russell A, Chung RJ, et al. A "novel" intervention: A pilot study of children's literature and healthy lifestyles. Pediatrics 2010; 125:e513-e517.

37. Cullen KW, Thompson D. Feasibility of an 8-week African American web-based pilot program promoting healthy eating behaviors: Family Eats. Am J Health Behav 2008; 32:40-51.

38. Trepka MJ, Newman FL, Davila EP, et al. Randomized controlled trial to determine the effectiveness of an interactive multimedia food safety education program for clients of the Special Supplemental Nutrition Program for Women, Infants, and Children. J Am Diet Assoc 2008; 108:978-984.

39. Baranowski T, Baranowski J, Cullen KW, et al. The Fun, Food, and Fitness Project (FFFP): The Baylor GEMS Pilot Study. Ethn Dis 2003; 13:S30-S39.

40. Armijo-Olivo S, Stiles CR, Hagen NA, et al. Assessment of study quality for systematic reviews: A comparison of the Cochrane Collaboration Risk of Bias Tool and the Effective Public Health Practice Project Quality Assessment Tool: Methodological research. J Eval Clin Pract 2012; 18:12-18.

41. Spook J, Paulussen T, Kok G, et al. Evaluation of a serious self-regulation game intervention for overweightrelated behaviors ("Balance It"): A pilot study. J Med Internet Res 2016; 18:e225.

42. Silk KJ, Sherry J, Winn B, et al. Increasing nutrition literacy: Testing the effectiveness of print, web site, and game modalities. J Nutr Educ Behav 2008; 40:3-10.

43. Dias M, Agante L. Can advergames boost children's healthier eating habits? A comparison between healthy and non-healthy food. J Consumer Behav 2011; 10:152160.

44. Folkvord F, Anschutz DJ, Buijzen M, et al. The effect of playing advergames that promote energy-dense snacks or fruit on actual food intake among children. Am J Clin Nutr 2013; 97:239-245.

45. Thompson D, Bhatt R, Vazquez I, et al. Creating action plans in a serious video game increases and maintains child fruit-vegetable intake: A randomized controlled trial. Int $\mathbf{J}$ Behav Nutr Phys Act 2015; 12:39.

46. Folkvord F, van 't Riet J. The persuasive effect of advergames promoting unhealthy foods among children: A meta-analysis. Appetite 2018; 129:245-251.

47. Duncan LR, Hieftje KD, Culyba S, et al. Game playbooks: Tools to guide multidisciplinary teams in developing videogame-based behavior change interventions. Transl Behav Med 2014; 4:108-116.

48. Elliot DL, Goldberg L, MacKinnon DP, et al. Empiric validation of a process for behavior change. Transl Behav Med 2016; 6:449-456.

49. Folkvord F, Anschutz DJ, Emma B, et al. Food advertising and eating behavior in children. Curr Opin Behav Sci 2016; 9:26-31.

50. Thompson D, Baranowski T, Buday R, et al. Serious video games for health: How behavioral science guided the development of a serious video game. Simul Gaming 2010; 41:587-606.

51. Baranowski T, Baranowski J, Thompson D, et al. Behavioral science in video games for children's diet and physical activity change: Key research needs. J Diabetes Sci Technol 2011; 5:229-233.

52. Perski O, Blandford A, West R, et al. Conceptualising engagement with digital behaviour change interventions: A systematic review using principles from critical interpretive synthesis. Transl Behav Med 2017; 7:254-267.

53. Bjork S, Holopainen J. Patterns in Game Design. Rockland, MA: Charles River Media; 2004.

54. Lucero A, Karapanos E, Arrasvuori J, et al. Playful or gameful? Creating delightful user experiences. Interactions 2014; 21:34-39.

55. Baranowski T, Jago R. Understanding the mechanisms of change in children's physical activity programs. Exerc Sport Sci Rev 2005; 33:163-168.

56. Michie S, Richardson M, Johnston M, et al. The Behavior Change Technique Taxonomy (v1) of 93 hierarchically clustered techniques: Building an international consensus for the reporting of behavior change interventions. Ann Behav Med 2013; 46:81-95.

57. Michie S, Wood CE, Johnston M, et al. Behaviour change techniques: The development and evaluation of a taxonomic method for reporting and describing behaviour change interventions (a suite of five studies involving consensus methods, randomised controlled trials and analysis of qualitative data). Health Technol Assess 2015; 19:1-188.

58. Simons M, Baranowski J, Thompson D, et al. Child goal setting of dietary and physical activity in a serious videogame. Games Health J 2013; 2:150-157.

59. Buday R, Baranowski T, Thompson D. Fun and games and boredom. Games Health J 2012; 1:257-261.

60. Leung MM, Tripicchio G, Agaronov A, et al. Manga comic influences snack selection in Black and Hispanic New York City youth. J Nutr Educ Behav 2014; 46:142147.

61. Crutzen R, van 't Riet J, Short CE. Enjoyment: A conceptual exploration and overview of experimental evidence in the context of games for health. Games Health J 2016; 5:15-20.

62. Marsh T, Xuejin C, Nickole LZ, et al. Fun and learning: The power of narrative. In: 6th International Conference on Foundations of Digital Games, June 28-July 01, 2011. Bordeaux, France: ACM; 2011, pp. 23-29. 
63. Bass M, Morris S, Neapolitan R. Utilizing multidimensional computer adaptive testing to mitigate burden with patient reported outcomes. AMIA Annu Symp Proc 2015; 2015:320-328.

64. Baranowski T, Lytle L. Should the IDEFICS outcomes have been expected? Obes Rev 2015; 16(Suppl 2):162172.

65. Li B, Liu WJ, Cheng KK, et al. Development of the theory-based Chinese primary school children physical activity and dietary behaviour changes intervention (CHIRPY DRAGON): Development of a cluster-randomised controlled trial. Lancet 2016; 388(Suppl 1):S51.

66. Baranowski T, Baranowski J, Thompson D, et al. Video game play, child diet, and physical activity behavior change: A randomized clinical trial. Am J Prev Med 2011; 40:33-38.

67. Baranowski T, Baranowski J, Cullen KW, et al. Squire's Quest! Dietary outcome evaluation of a multimedia game. Am J Prev Med 2003; 24:52-61.

68. Baranowski T, O'Connor T, Hughes S, et al. Smart phone video game simulation of parent-child interaction: Learning skills for effective vegetable parenting. In: Arnab S, Dunwell I, Debattista K (eds.). Serious Games for Healthcare: Applications and Implications. Hershey, PA: IGI Global; 2012, pp. 248-265.

69. Sneed JR, Rutherford BR, Rindskopf D, et al. Design makes a difference: A meta-analysis of antidepressant response rates in placebo-controlled versus comparator trials in late-life depression. Am J Geriatr Psychiatry 2008; 16: $65-73$.

70. Kunz R, Vist G, Oxman AD. Randomisation to protect against selection bias in healthcare trials. Cochrane Database Syst Rev 2007:MR000012.

71. Pildal J, Hrobjartsson A, Jorgensen KJ, et al. Impact of allocation concealment on conclusions drawn from metaanalyses of randomized trials. Int J Epidemiol 2007; 36: 847-857.

72. Schulz KF, Chalmers I, Hayes RJ, et al. Empirical evidence of bias. Dimensions of methodological quality associated with estimates of treatment effects in controlled trials. JAMA 1995; 273:408-412.

73. Chalmers TC, Celano P, Sacks HS, et al. Bias in treatment assignment in controlled clinical trials. $\mathrm{N}$ Engl $\mathrm{J}$ Med 1983; 309:1358-1361.

74. Moher D, Cook DJ, Jadad AR, et al. Assessing the quality of reports of randomised trials: Implications for the conduct of meta-analyses. Health Technol Assess 1999; 3:iiv, $1-98$.

75. Armijo-Olivo S, Macedo LG, Gadotti IC, et al. Scales to assess the quality of randomized controlled trials: A systematic review. Phys Ther 2008; 88:156-175.

76. Khan KS, ter Riet G, Popay J, et al. Stage II: Conducting the review, phase 5: Study quality assessment. In: Khan KS, ter Riet G, Glanville J (eds.). Undertaking Systematic Reviews of Research Effectiveness: CRD's Guidance for Those Carrying Out or Commissioning Reviews, 2nd ed. York, United Kingdom: NHS Centre for Reviews and Dissemination, University of York; 2001, pp. 1-20.

77. Balshem H, Helfand M, Schunemann HJ, et al. GRADE guidelines: 3. Rating the quality of evidence. J Clin Epidemiol 2011; 64:401-406.

78. Baranowski T, Blumberg F, Buday R, et al. Games for health for children-Current status and needed research. Games Health J 2016; 5:1-12.
79. Baranowski T. Games for health research-Past, present, and future. Präv Gesundheitsf 2018; https://doi.org/10.1007/ s11553-018-0657-y

80. Kirkpatrick SI, Baranowski T, Subar AF, et al. Conducting and interpreting validations of dietary assessment methods: Concepts, considerations and recommendations. J Acad Nutr Diet 2018; submitted.

81. Spruijt-Metz D, Wen CK, Bell BM, et al. Advances and controversies in diet and physical activity measurement in youth. Am J Prev Med 2018; 55:e81-e91.

82. Baranowski T, Blumberg F, Gao Z, et al. Getting research on games for health funded. Games Health J 2017; 6:1-8.

83. Brown AW, Ioannidis JP, Cope MB, et al. Unscientific beliefs about scientific topics in nutrition. Adv Nutr 2014; 5:563-565.

84. George BJ, Beasley TM, Brown AW, et al. Common scientific and statistical errors in obesity research. Obesity (Silver Spring) 2016; 24:781-790.

85. Munafò MR, Nosek BA, Bishop DVM, et al. A manifesto for reproducible science. Nat Hum Behav 2017; 1:0021.

86. Turnin MC, Tauber MT, Couvaras O, et al. Evaluation of microcomputer nutritional teaching games in 1,876 children at school. Diabetes Metab 2001; 27:459-464.

87. Serrano EL, Anderson JE. The evaluation of food pyramid games, a bilingual computer nutrition education program for Latino youth. J Fam Consum Sci Educ 2004; 22:1-16.

88. Peng W. Design and evaluation of a computer game to promote a healthy diet for young adults. Health Commun 2009; 24:115-127.

89. Baños RM, Cebolla A, Oliver E, et al. Efficacy and acceptability of an Internet platform to improve the learning of nutritional knowledge in children: The ETIOBE Mates. Health Educ Res 2013; 28:234-248.

90. Schneider KL, Ferrara J, Lance B, et al. Acceptability of an online health videogame to improve diet and physical activity in elementary school students: "Fitter Critters". Games Health J 2012; 1:262-268.

91. Majumdar D, Koch PA, Lee H, et al. "Creature-101”: A serious game to promote energy balance-related behaviors among middle school adolescents. Games Health J 2013; 2:280-290.

92. Folkvord F, Anschutz DJ, Nederkoorn C, et al. Impulsivity, "advergames," and food intake. Pediatrics 2014; 133:1007-1012.

93. Johnson-Glenberg MC, Savio-Ramos C, Henry H. "Alien Health": A nutrition instruction exergame using the Kinect sensor. Games Health J 2014; 3:241-251.

94. Jones BA, Madden GJ, Wengreen HJ. The FIT Game: Preliminary evaluation of a gamification approach to increasing fruit and vegetable consumption in school. Prev Med 2014; 68:76-79.

95. Rosi A, Scazzina F, Ingrosso L, et al. The "5 a day" game: A nutritional intervention utilising innovative methodologies with primary school children. Int $\mathrm{J}$ Food Sci Nutr 2015; 66:713-717.

96. Sharma SV, Shegog R, Chow J, et al. Effects of the Quest to Lava Mountain computer game on dietary and physical activity behaviors of elementary school children: A pilot group-randomized controlled trial. J Acad Nutr Diet 2015; 115:1260-1271.

97. Shiyko M, Hallinan S, Seif El-Nasr M, et al. Effects of playing a serious computer game on body mass index and nutrition knowledge in women. JMIR Serious Games 2016; 4:e8. 
98. Fraticelli F, Marchetti D, Polcini F, et al. Technologybased intervention for healthy lifestyle promotion in Italian adolescents. Ann Ist Super Sanita 2016; 52:123-127.

99. Joyner D, Wengreen HJ, Aguilar SS, et al. The FIT Game III: Reducing the operating expenses of a game-based approach to increasing healthy eating in elementary schools. Games Health J 2017; 6:111-118.

100. Wang JJ, Baranowski T, Lau PW, et al. Story immersion may be effective in promoting diet and physical activity in Chinese children. J Nutr Educ Behav 2017; 49:321-329 e1.
Address correspondence to:

Tom Baranowski, PhD

Department of Pediatrics

USDA/ARS Children's Nutrition Research Center

Baylor College of Medicine

1100 Bates Street

Houston, TX 77030

E-mail: tbaranow@bcm.edu 